\title{
Influência da Hipertrofia Mamária na Capacidade Funcional das Mulheres
}

\section{Influence of Breast Hypertrophy in the Women's Functional Capacity}

\author{
Carlos Delano Mundim Araújo ${ }^{(1)}$, Heitor Carvalho Gomes $^{(2)}$, Daniela Francescato Veiga ${ }^{(2)}$,
} Bernardo Hochman $^{(2)}$, Paulo Magalhães Fernandes ${ }^{(1)}$, Neil Ferreira Novo ${ }^{(3)}$, Lydia Masako Ferreira ${ }^{(4)}$

\section{RESUMO}

Objetivo: investigar a influência da hipertrofia mamária no sistema musculoesquelético por meio da avaliação da capacidade funcional das mulheres. Método: foram avaliadas 50 mulheres com hipertrofia mamária, e os resultados foram comparados com os de 50 mulheres com mamas normais. Para avaliação da capacidade funcional, utilizou-se o STANFORD HEALTH ASSESSMENT QUESTIONNAIRE 20 (HAQ-20), que consiste de 20 questões sobre atividades do cotidiano em que a entrevistada tem quatro opções de resposta, que são pontuadas. Quanto menor a pontuação, melhor a capacidade funcional. Resultados: a média dos escores do grupo-controle foi 0,10 e do grupo-hipertrofia foi igual a 0,71. Esses escores submetidos ao teste de Mann-Whitney mostraram que o grupo-hipertrofia apresentou valores significantemente maiores do que o grupo-controle $(\mathrm{p}<0,00)$. Conclusão: mulheres com hipertrofia mamária apresentam menor capacidade funcional, ou seja, mais dificuldade para realizar atividades comuns da vida diária.

Palavras-chave: mama, questionários, postura, hipertrofia, dor nas costas.

\section{INTRODUÇÃO}

O termo capacidade funcional tem sido usado como um dos parâmetros de avaliação de qualidade de vida relacionado à saúde. É interpretado como a capacidade de o paciente realizar atividades diárias comuns ${ }^{(1)}$. A hipertrofia mamária pode ser definida como "um aumento da glândula mamária além dos limites fisiológicos, com exceção dos aumentos causados por ferimentos, hemorragias, inflamações e gravidez"(2). Estudos demonstraram o quanto a hipertrofia mamária pode alterar o componente funcional, acarretando transtornos de ordem circulatória, respiratória e postural.

\section{ABSTRACT}

Objective: to investigate the influence of breast hypertrophy in the musculoskeletal-system through the evaluation of the functional capacity in women. Method: 50 women with breast hypertrophy were studied and the results were compared to the 50 women without breast hypertrophy. The STANFORD HEALTH ASSESSMENT QUESTIONNAIRE 20 (HAQ-20) was applied to assess the functional capacity. It contains 20 questions about daily activities in which the patient has four answer options, which are scored. Lower scores indicate better functional capacity. Results: the mean score in the control group was 0.10 , while in the breast hypertrophy group it was 0.71 . When Mann-Whitney test was run, hypertrophy group showed statistically higher scores $(p<0.00)$. Conclusion: breast hypertrophy patients have more difficulties to perform common activities of the daily life.

Keywords: breast, questionnaires, posture, hypertrophy, back pain.

Isso traz repercussão no centro de gravidade normal e exacerbação das curvaturas fisiológicas da coluna vertebral, ocasionando dor, alterações posturais da região deltóidea e dermatite submamária ${ }^{(3-5)}$. Por isso, alterações mamárias do tamanho têm sido tratadas como anomalias ${ }^{(4)}$.

As dorsolombalgias têm alta prevalência e representam um desafio para a medicina em geral, sendo as mais freqüentes as de origem lombar. Interferem na vida das pessoas, causando limitação funcional e custos elevados com a saúde ${ }^{(6)}$. A dor aguda de coluna costuma ocorrer em torno dos 25 anos de idade e, em 90\% dos casos, desaparece em

Recebido em 17/01/07. Aprovado, após revisão, em 24/03/07.

Trabalho realizado no Programa de Mestrado Interinstitucional (Minter) Unifesp/Univás.

1. Médicos ortopedistas da Universidade do Vale do Sapucaí (UNIVÁS), Pouso Alegre (MG), Aluno do Programa de Mestrado Interinstitucional (Minter) Unifesp/Univás.

2. Professores doutores da Disciplina de Cirurgia Plástica da Unifesp

3. Professor-adjunto do Departamento de Bioestatística da Unifesp

4. Professora titular da Disciplina de Cirurgia Plástica e coordenadora do Programa de Pós-Graduação em Cirurgia Plástica da Unifesp.

Endereço para correspondência: Carlos Delano Mundim Araújo, Av. Getúlio Vargas, 79, CEP 37550-000, Pouso Alegre, MG, Brasil,

Telefax: (35) 3423-8700/(35) 3422-5830, celular: (35) 8833-0297, e-mail: carlosdm@uai.com.br 
30 dias com ou sem tratamento, sendo que, se os possíveis fatores causais não forem afastados, o risco de recorrência é de $60 \%$ no mesmo ano ou no máximo em dois anos ${ }^{(7)}$. Nesse sentido, a hipertrofia mamária também tem sido citada como causa relevante de dor na coluna ${ }^{(8)}$.

Desde a década de 1940, surgiram nos EUA diversos questionários utilizados como instrumentos de avaliação de qualidade de vida, transformando avaliações subjetivas em objetivas. Nos últimos 20 anos, a avaliação da forma de percepção da doença pelo paciente tem sido objeto de muitos estudos. Pesquisadores têm se preocupado não somente com os aspectos biológicos da doença, mas também com suas conseqüências em vários aspectos da vida das pacientes ${ }^{(1)}$.

O objetivo deste estudo foi verificar a influência da hipertrofia mamária no sistema musculoesquelético por meio de um questionário de avaliação da capacidade funcional das pacientes.

\section{PACIENTES E MÉTODOS}

Trata-se de estudo primário, observacional, transversal, analítico, controlado, em seres humanos.

Foram selecionadas, consecutivamente, 50 pacientes com hipertrofia mamária (grupo-estudo) e $\mathbf{5 0}$ pacientes com mamas normais (grupo-controle), com idade entre 18 e 59 anos, dos ambulatórios de Cirurgia Plástica e Ortopedia e Traumatologia do Hospital das Clínicas Samuel Libânio (HCSL).

Foram incluídas no grupo-estudo as mulheres com hipertrofia mamária, de acordo com o índice de Sacchini ${ }^{(9)}$ e, no grupo-controle, as mulheres com mamas normais.

Foram excluídas mulheres com gestação ou amamentação há menos de um ano, doenças sistêmicas não-controladas, doenças agudas, assimetria mamária, operação prévia nas mamas ou coluna, além de índice de massa corporal $<18,5 \mathrm{~kg} / \mathrm{m}^{2}$ (IMC com baixo peso) ou $\geq 30 \mathrm{~kg} / \mathrm{m}^{2}$ (IMC com obesidade).

Todas as pacientes assinaram o termo de consentimento livre e esclarecido. Este estudo foi aprovado pelo Comitê de Ética em Pesquisa da Univás.

As pacientes foram submetidas à coleta de dados sociodemográficos, medição das mamas, aferição do peso corporal, além da medida da estatura em metros. Em seguida, responderam ao questionário de avaliação da capacidade funcional STANFORD HEALTH ASSESSMENT QUESTIONNAIRE 20 (HAQ-20), sob a forma de entrevistas.
A mensuração da hipertrofia mamária foi feita de forma objetiva pelo índice de Sacchini, com a paciente em posição ortostática com fita métrica milimetrada. Sacchini et al. ${ }^{(9)}$ utilizaram a média entre duas distâncias para classificar o tamanho das mamas, individualmente (Figura 1): entre a papila mamária e o sulco mamário e entre a papila mamária e a margem lateral do esterno. A mama é classificada em: 1) pequena ou hipomastia = média menor do que $9 \mathrm{~cm}$; 2) média ou normal = média entre $9 \mathrm{~cm}$ e $11 \mathrm{~cm}$; e 3) grande ou hipertrofia = média maior do que $11 \mathrm{~cm}$.

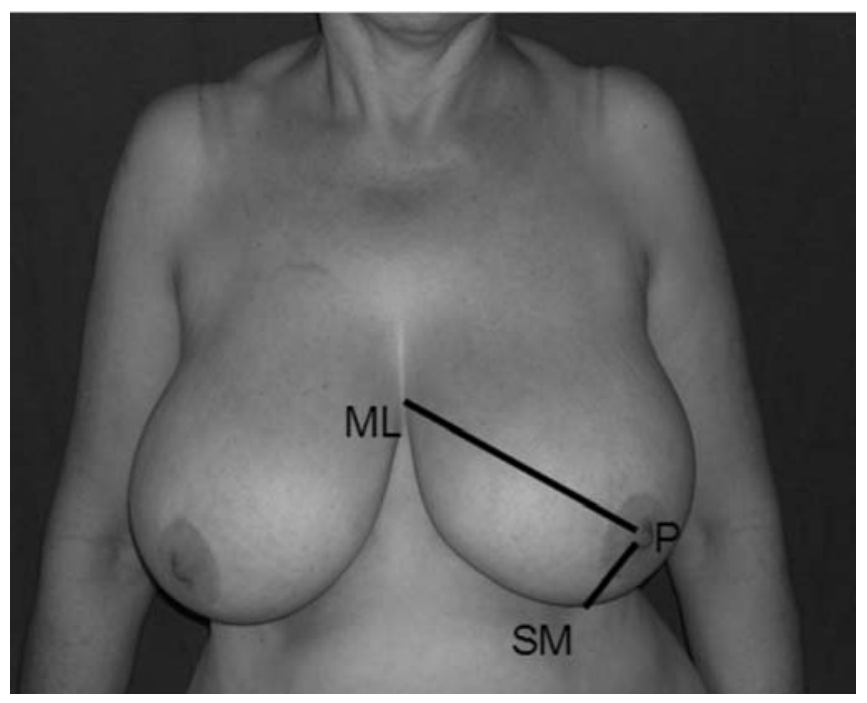

Figura 1 - Medida do índice de Sacchini.

$\mathrm{ML}=$ margem lateral do esterno; $\mathrm{P}=$ papila mamária; $\mathrm{SM}=$ sulco mamário

De acordo com esses autores, é considerada assimetria mamária a diferença entre as médias das duas mamas superior a $2 \mathrm{~cm}$.

Para avaliação da capacidade funcional, foi utilizado o HAQ-20 (Quadro 1), que foi traduzido, adaptado e validado para uso no Brasil ${ }^{(10)}$. Consiste em 20 questões sobre atividades de vida diária, que são pontuadas conforme a capacidade de realizá-las. As questões são divididas em oito componentes, e é feita a média aritmética apenas do maior escore de cada componente (Quadro 2). Os pontos variam de 0 a 3 . Quanto menor a pontuação, melhor a capacidade funcional.

Método estatístico: para análise dos resultados, foi aplicado o teste de Mann-Whitney, para comparar a idade e os escores do HAQ-20 entre os dois grupos. Teste " $t$ " de Student para comparar o IMC. Teste do qui-quadrado com a finalidade de comparar os grupos em relação às freqüências dos oito componentes do HAQ-20. Fixou-se em 0,05 o nível de rejeição da hipótese da nulidade. 
QUADRO 1

VERSÃo BRASILEIRA DO QUESTIONÁRIO DE CAPACIDADE FUNCIONAL HAQ-20

\begin{tabular}{|c|c|c|c|c|c|}
\hline & & $\begin{array}{c}\text { Sem } \\
\text { dificuldade }\end{array}$ & $\begin{array}{l}\text { Com alguma } \\
\text { dificuldade }\end{array}$ & $\begin{array}{l}\text { Com muita } \\
\text { dificuldade }\end{array}$ & $\begin{array}{l}\text { Incapaz } \\
\text { de fazer }\end{array}$ \\
\hline 01 & Vestir-se, inclusive amarrar os cordões dos seus sapatos, abotoar as suas roupas? & 0 & 1 & 2 & 3 \\
\hline 02 & Lavar a sua cabeça e os seus cabelos? & 0 & 1 & 2 & 3 \\
\hline 03 & Levantar-se de uma maneira ereta de uma cadeira de encosto reto e sem braços? & 0 & 1 & 2 & 3 \\
\hline 04 & Deitar-se e levantar-se da cama? & 0 & 1 & 2 & 3 \\
\hline 05 & Cortar um pedaço de carne? & 0 & 1 & 2 & 3 \\
\hline 06 & Levar à boca um copo ou uma xícara cheia de café, leite ou água? & 0 & 1 & 2 & 3 \\
\hline 07 & Abrir um saco de leite comum? & 0 & 1 & 2 & 3 \\
\hline 08 & Caminhar em lugares planos? & 0 & 1 & 2 & 3 \\
\hline 09 & Subir cinco degraus? & 0 & 1 & 2 & 3 \\
\hline 10 & Lavar seu corpo inteiro e secá-lo após o banho? & 0 & 1 & 2 & 3 \\
\hline 11 & Tomar um banho de chuveiro? & 0 & 1 & 2 & 3 \\
\hline 12 & Sentar-se e levantar-se de um vaso sanitário? & 0 & 1 & 2 & 3 \\
\hline 13 & $\begin{array}{l}\text { Levantar os braços e pegar um objeto de mais ou menos } 2,5 \text { quilos, } \\
\text { que está posicionado um pouco acima de sua cabeça? }\end{array}$ & 0 & 1 & 2 & 3 \\
\hline 14 & Curvar-se para pegar suas roupas no chão? & 0 & 1 & 2 & 3 \\
\hline 15 & Segurar-se em pé no ônibus ou no metrô? & 0 & 1 & 2 & 3 \\
\hline 16 & Abrir potes ou vidros de conserva que tenham sido previamente abertos? & 0 & 1 & 2 & 3 \\
\hline 17 & Abrir e fechar torneiras? & 0 & 1 & 2 & 3 \\
\hline 18 & Fazer compras na redondeza onde mora? & 0 & 1 & 2 & 3 \\
\hline 19 & Entrar e sair de um ônibus? & 0 & 1 & 2 & 3 \\
\hline 20 & Realizar tarefas tais como usar a vassoura para varrer e o rodo para puxar água? & 0 & 1 & 2 & 3 \\
\hline
\end{tabular}

QuAdro 2

Avaliação dos ESCORES do HAQ-20

\begin{tabular}{|c|c|c|c|}
\hline $\begin{array}{l}\text { Componente } 1 \rightarrow \\
\text { (vestir-se) }\end{array}$ & Perguntas 1 e 2 & $\rightarrow$ & Maior escore $=$ \\
\hline $\begin{array}{l}\text { Componente } 2 \rightarrow \\
\text { (levantar-se) }\end{array}$ & Perguntas 3 e 4 & $\rightarrow$ & Maior escore $=$ \\
\hline $\begin{array}{l}\text { Componente } 3 \rightarrow \\
\text { (alimentar-se) }\end{array}$ & Perguntas 5,6 e 7 & $\rightarrow$ & Maior escore $=$ \\
\hline $\begin{array}{l}\text { Componente } 4 \rightarrow \\
\text { (caminhar) }\end{array}$ & Perguntas 8 e 9 & $\rightarrow$ & Maior escore $=$ \\
\hline $\begin{array}{l}\text { Componente } 5 \rightarrow \\
\text { (higiene pessoal) }\end{array}$ & Perguntas 10,11 e 12 & $\rightarrow$ & Maior escore $=$ \\
\hline $\begin{array}{l}\text { Componente } 6 \rightarrow \\
\text { (alcançar objetos) }\end{array}$ & Perguntas 13 e 14 & $\rightarrow$ & Maior escore $=$ \\
\hline $\begin{array}{l}\text { Componente } 7 \rightarrow \\
\text { (apreender objetos) }\end{array}$ & Perguntas 15,16 e 17 & $\rightarrow$ & Maior escore $=$ \\
\hline \multirow[t]{2}{*}{$\begin{array}{l}\text { Componente } 8 \rightarrow \\
\text { (outras atividades) }\end{array}$} & Perguntas 18,19 e 20 & $\rightarrow$ & Maior escore $=$ \\
\hline & & & $\begin{array}{l}\text { Média aritmética dos } \\
\text { escores dos componentes }=\end{array}$ \\
\hline
\end{tabular}

\section{RESULTADOS}

A média de idade encontrada no grupo-controle foi de 32,72 ( \pm 11$)$ anos e no grupo-estudo foi de 32,20 $( \pm 8)$ anos. No grupo-controle, a média do IMC foi de $22,27 \mathrm{~kg} / \mathrm{m}^{2}( \pm 2,28)$ e, no grupo-estudo, a média foi de $25,88 \mathrm{~kg} / \mathrm{m}^{2}( \pm 2,58)$. A distribuição de cor e escolaridade pode ser vista nas Tabelas 1 e 2 , respectivamente.
TABELA 1

Pacientes DO GRUPO-ESTUdO E DO GRUPO-CONTROLE SEGUNDO A COR

\begin{tabular}{|c|c|c|c|c|c|}
\hline \multirow[b]{2}{*}{ Cor } & & \multicolumn{2}{|c|}{ Grupo } & \multirow[b]{2}{*}{ Total } & \multirow[b]{2}{*}{$p$} \\
\hline & & Controle & Estudo & & \\
\hline \multirow[t]{2}{*}{ Não-branca } & $n$ & 18 & 6 & 24 & \\
\hline & $\%$ & 36,0 & 12 & 24 & \\
\hline \multirow[t]{2}{*}{ Branca } & $\mathrm{n}$ & 32 & 44 & 76 & 0,00 * \\
\hline & $\%$ & 64 & 88 & 76 & \\
\hline \multirow[t]{2}{*}{ TOTAL } & $\mathrm{n}$ & 50 & 50 & 50 & \\
\hline & $\%$ & 100 & 100 & 100 & \\
\hline
\end{tabular}

* Significância estatística (teste do qui-quadrado)

TABELA 2

GRAU DE INSTRUÇÃO DOS GRUPOS

\begin{tabular}{|c|c|c|c|c|c|}
\hline \multirow[b]{2}{*}{ Ensino } & \multicolumn{3}{|c|}{ Grupo } & \multirow[b]{2}{*}{ Total } & \multirow[b]{2}{*}{$\mathrm{p}$} \\
\hline & & Controle & Estudo & & \\
\hline \multirow[t]{2}{*}{ Fundamental } & $\mathrm{n}$ & 5 & 2 & 7 & \\
\hline & $\%$ & 10 & 4 & 7 & \\
\hline \multirow[t]{2}{*}{ Médio } & $\mathrm{n}$ & 22 & 37 & 59 & \\
\hline & $\%$ & 44 & 74 & 59 & 0,00 * \\
\hline \multirow[t]{2}{*}{ Superior } & $\mathrm{n}$ & 23 & 11 & 34 & \\
\hline & $\%$ & 46 & 22 & 34 & \\
\hline Total (n) & & 50 & 50 & 100 & \\
\hline
\end{tabular}

* Significância estatística (teste do qui-quadrado)

As porcentagens de respostas dos componentes do HAQ-20 dos grupos podem ser observadas nos Gráficos 1 e 2.

Os resultados do HAQ-20 (Tabela 3) mostram que pacientes com hipertrofia mamária apresentam a capacidade funcional diminuída. 
GRÁFICO 1

Porcentagem de REsPostas DO GRUPO-CONTROLE NOS COMPONENTES DO HAQ-20

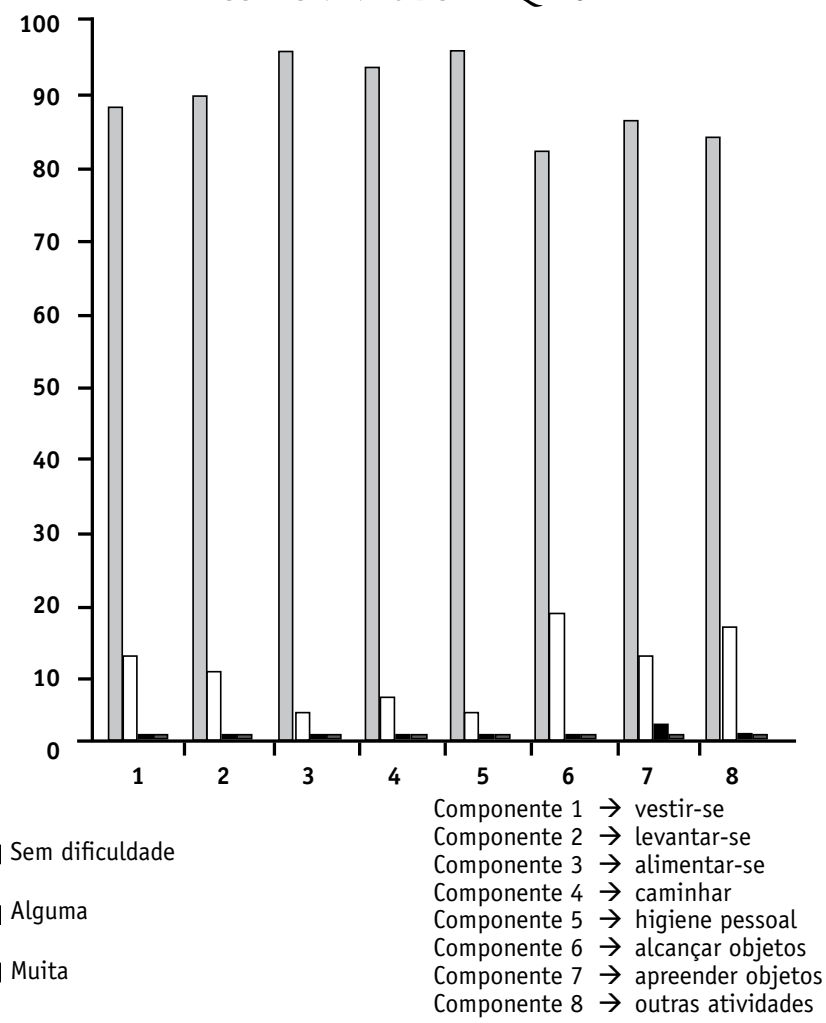

GrÁFICO 2

Porcentagem DE RESPOSTAS DO GRUPO-ESTUdO NOS

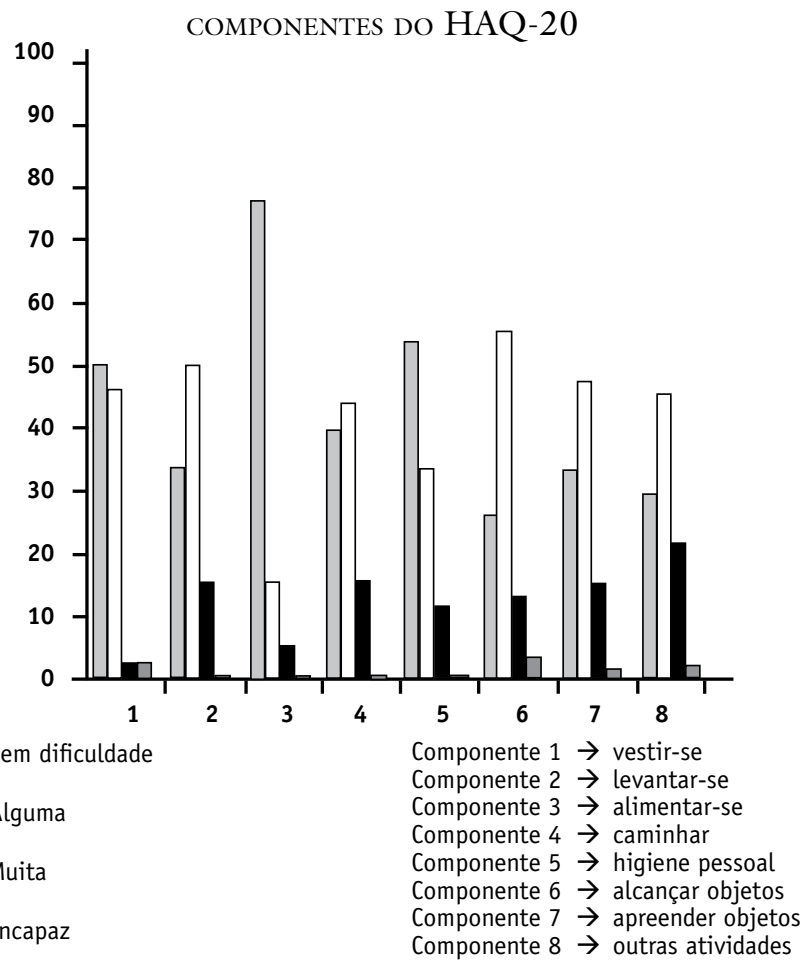

TABELA 3

ESCALA DO HAQ-20 DOS GRUPOS

\begin{tabular}{cc|c|c|c|c|c|c|c|c}
\hline & Grupo & N & Média & DP & Mediana & Mínimo & Máximo & $\mathbf{p}$ & $\mathbf{z}$ \\
\hline \multirow{4}{*}{ HAQ-20 } & Controle & 50 & 0,10 & 0,21 & 0,00 & 0,00 & 2,00 & & \\
& Estudo & 50 & 0,71 & 0,52 & 0,62 & 0,00 & 2,00 & $\mathbf{0}, \mathbf{0 0}$ & $\mathbf{6 , 2 0 *}$ \\
& Total & 100 & 0,39 & & 0,15 & 0,00 & 2,00 & & \\
\hline
\end{tabular}

* Significância estatística (teste de Mann-Whitney).

\section{DISCUSSÃO}

Vários trabalhos avaliaram a capacidade funcional em mulheres com hipertrofia mamária por meio de outros instrumentos e comprovaram um alto percentual de inaptidão ao trabalho com piora das atividades físicas, como tarefas domésticas e brincadeiras com crianças ${ }^{(11-14)}$. O único estudo anterior envolvendo capacidade funcional e hipertrofia mamária utilizando-se o HAQ-20 foi o de Freire ${ }^{(15)}$, que também encontrou diminuição da capacidade funcional.

No presente estudo, quanto ao IMC encontrado no grupo-estudo (média com sobrepeso), houve concordância com estudo prévio ${ }^{(16)}$ que mostrou que somente $30 \%$ das pacientes com IMC normal procuram por mamoplastia redutora. Outros estudos envolvendo questionários e sintomas da hipertrofia mamária ${ }^{(8,14)}$ também encontraram uma tendência para média elevada do IMC, confirmando a observação clínica de que as mulheres que mais procuram mamoplastia redutora estão com IMC acima de $25 \mathrm{~kg} / \mathrm{m}^{2}$.

Em relação à cor, não se observou homogeneidade entre os dois grupos estudados. Entretanto, não foram observados, na literatura, estudos envolvendo instrumento semelhante em que se relatasse influência da cor da pele na freqüência da hipertrofia mamária ou em seus sintomas.

Os escores do HAQ-20 encontrados no grupo-estudo, superiores ao grupo-controle, evidenciam uma maior influência da hipertrofia mamária no sistema musculoesquelético dessas pacientes. Isso pode ser visto como uma maior dificuldade na realização das suas funções diárias. A diminuição da capacidade funcional nas pacientes com hipertrofia mamária também pode ser inferida a uma maior dependência física e mental, diminuição da capacidade do trabalho, bem como o aumento do uso de serviços de saúde ${ }^{(17)}$. Sendo assim, as pacientes com diminuição da capacidade funcional por hipertrofia mamária têm maior possibilidade de alívio dos sintomas com uma mamoplastia redutora $^{(18)}$ e também de uma melhora da sua qualidade de vida de forma global.

Analisando os domínios do HAQ-20 do presente estudo, as menores dificuldades encontradas pelas pacientes com hipertrofia foram relacionadas com seus cuidados pes- 
soais (alimentar-se, higiene pessoal e vestir-se). Nenhuma paciente relatou ser incapaz de levantar-se, alimentar-se, caminhar ou de realizar sua higiene pessoal. A maior dificuldade encontrada foi no componente 6 (alcançar objetos).

Freire $^{(15)}$, que também utilizou o HAQ-20 em mulheres com hipertrofia mamária, encontrou menor dificuldade no componente 3 (alimentar-se) como neste trabalho, mas a maior dificuldade encontrada foi no componente 5 (higiene pessoal).

Além do aspecto estético, a principal queixa das pacientes com hipertrofia mamária é dor no sistema musculoesquelético ${ }^{(19)}$. Sintomas que vão desde síndrome do túnel do carpo até dor na coluna e diminuição da capacidade funcional apresentam melhora com a mamoplastia redutora ${ }^{(20,21)}$.

Freqüentemente os profissionais vêem-se pressionados pelos convênios médicos e pela paciente. Por um lado, os planos de saúde ou seguradoras consideram o procedimento estético, o que, contratualmente, impede a mamoplastia redutora $^{(22)}$. Por outro lado, as pacientes relatam queixas que elas próprias relacionam à hipertrofia, seja pela real necessidade da operação ou como forma alternativa (nãocontratual) de conseguir o procedimento. O desejo da paciente em realizar a mamoplastia pode ter motivos óbvios e incontestáveis, mas existe uma linha tênue que separa a real necessidade do motivo puramente estético.

Apesar de vários estudos tratarem sobre a influência da hipertrofia mamária na postura e na coluna vertebral $^{(1,13,16,22,23)}$, na prática clínica, existe dificuldade para indicar a mamoplastia redutora sem finalidade estética, dada a subjetividade dos sintomas, bem como a escassez de parâmetros objetivos. Essa escassez faz com que os planos de saúde ou mesmo o Sistema Único de Saúde (SUS) tenham uma tendência a não permitir a realização do procedimento e continuem a duvidar da necessidade médica da mamoplastia redutora em pacientes sintomáticas.

Nos últimos anos, entre os mais importantes avanços da medicina está o consenso crescente sobre a importância da opinião do paciente sobre os resultados das intervenções

\section{REFERÊNCIAS}

1. Ware JE, Sherbourne CD: The MOS 36-item short-form health survey. Med Care 30(6): 473-83, 1992.

2. Foged J: Symptolatologien ved mamma hypertrofi. UgesKr Laeger 115(12): 439-51, 1953.

3. Birtchnell S, Whitfield P, Lacey JH: Motivational factors in women requesting augmentation and reduction mammaplasty. J Psychosom Res 34(5): 509-14, 1990. médicas ${ }^{(1)}$. Os índices, instrumentos ou questionários para avaliar a qualidade de vida ou seus componentes, como auto-estima e capacidade funcional, são formas de avaliar objetivamente situações consideradas subjetivas e têm sido usados em todo o mundo como maneira de analisar e comparar esses dados.

A avaliação da capacidade funcional pode se tornar uma referência adicional para a indicação da mamoplastia redutora. Neste estudo, em nenhum momento a paciente teve a promessa de que sua participação no estudo favoreceria de alguma maneira a realização da mamoplastia redutora.

A avaliação da capacidade funcional na hipertrofia mamária traz perspectivas para outras correlações, como a análise de alterações radiológicas ${ }^{(24)}$ com a hipertrofia. O surgimento de critérios objetivos trará sustentação ao profissional para a indicação não-estética da mamoplastia redutora e credibilidade à paciente para a liberação do procedimento por seu plano de saúde ou pelo SUS.

O questionário de avaliação da capacidade funcional mostrou-se exeqüível e prático de ser utilizado como rotina nas consultas médicas envolvendo queixas de hipertrofia mamária. A administração dos questionários por entrevistas é um método mais apropriado em estudos envolvendo pacientes que tiveram acesso apenas ao ensino fundamental ${ }^{(25)}$, pois estes apresentam significativo índice de perda de dados por erro na resolução dos questionários auto-administrados.

A avaliação da capacidade funcional na hipertrofia mamária também permite auxiliar o quanto o investimento nas mamoplastias redutoras poderia trazer de benefícios e colaborar em tomadas de decisão quanto à distribuição de verbas nos sistemas de saúde, público e privado.

\section{CONCLUSÃO}

A capacidade funcional nas pacientes com hipertrofia mamária encontra-se diminuída.

Declaramos a inexistência de conflitos de interesse.
4. Hollyman JA, Lacey JH, Whitfield PJ, Wilson JSP: Surgery for the psyche: A longitudinal study of women undergoing reduction mammoplasty. Br J Surg 39(2): 222-4, 1986.

5. Letterman G, Schurter M: The effects of mammary hypertrophy on skeletal system. Ann Plast Surg 5(6): 425-31, 1980.

6. Laat E: Estudo de episódios lombálgicos e condições de trabalho em militares. [Tese Mestrado]. Porto Alegre. Escola de Engenharia-UFRGS; 2005. 
7. Kelsey JL, Golden AL: Occupational and workplacefactors associated with low back pain. State of the art reviews: Occup Med 3(1): 7-16, 1988.

8. Gonzalez F, Walton RL, Shafer B, Matory Jr. WE, Borah GL: Reduction mammaplasty improves symptoms of macromastia. Plast Reconstr Surg 91(7): 1270-6, 1993.

9. Sacchini V, Luini A, Tana S, et al: Quantitative and qualitative cosmetic evaluation after conservative treatment for breast cancer. Eur J Cancer 27(11): 1395-400, 1991.

10. Ferraz MB, Oliveira LM, Araújo PMP, Atra E, Tugwell P: Crosscultural reliability of the physical ability dimension of the Health Assessment Questionnaire. J Rheumatol 17(6): 813-7, 1990.

11. Raispis T, Zehring RD, Downey DL: Long-term functional results after reduction mammaplasty. Plast Reconstr Surg 102: 2139-47, 1998.

12. Attrhem H, Holmner S, Janson P: Reduction mammaplasty: Symptoms, complications, and late results. Scand J Reconstr Hand Surg 32: 281-6, 1998.

13. Shakespeare V, Postle K: A qualitative study of patients, vies of effects of breast reduction surgery: a 2 year follow up survey. B J Plast Surg 52: 198-204, 1999.

14. Blomqvist L, Eriksson A, Brandberg Y: Reduction mammaplasty provides long term improvement in health status and quality of life. Plast Reconstr Surg 106: 991-7, 2000.

15. Freire MAMS: Capacidade funcional e dor após a mamoplastia redutora. [Tese Doutorado]. São Paulo: Universidade Federal de São Paulo - Escola Paulista de Medicina; 2004.

16. Shakespeare V, Cole RP: Measuring patient based outcomes in a plastic surgery service: Breast reduction surgical patients. Br J Plast Surg 50: 242-8, 1997.
17. Ferraz MB, Quaresma MR, Aquino LRL, Atra E, Tugwell P, Goldsmith H: Reability of pain scales in the assessment of literature and illiterate patients with rheumatoid arthritis. J Rheumatol 17: 1022-4, 1990.

18. Klassen A, Jenkinson C, Fitzpatrick R, Goodacre T: Patient health related quality of life before and after aesthetic surgery. Br J Plast Surg 49(7): 433-8, 1996.

19. Pernia LR, Nonel DN, Leeper JD, Miller HL: Carpal tunel syndrome in women undergoing reduction mammaplasty. Plast Reconst Surg 105: 1314-9, 2000.

20. Chadbourne EB, Zang S, Gordon MJ, et al: Clinical outcomes in reduction mammaplasty: A systematic review and meta-analysis of published studies. Mayo clin 76: 503-10, 2001.

21. Jones SA, Bain J: Review of data describing outcomes that are used to assess changes in quality of life after reduction mammaplasty. Plast Reconstr Surg 108: 62-7, 2001.

22. Seitchik MW: Reduction mammaplasty: Criteria for insurance coverage. Plast Reconstr Surg 95(6): 1029-32, 1995.

23. Netscher DT, Meade RA, Goodman CM, Brehm BJ, Friedman JD, Thornby J: Physical and psychosocial symptoms among 88 volunteers subjects compared with patients seeking plastic surgery procedures to the breast. Plast Reconst Surg 105: 2366-72, 2000.

24. Fouquet B, Goupille P, Rouif V, Mateu J, Valat JP: Breast hypertrophy and dorsolumbar spine. Prognostic influences of lumbar lordosis: preliminary results. Rev Rheum Mal Osteoartic 58(6): 454-7, 1991.

25. D'Amorim AB: Avaliação das formas auto-administradas dos questionários MHAQ e SF-12 em pacientes com doenças reumáticas [tese Mestrado]. Universidade Federal de São Paulo - Escola Paulista de Medicina; 2001. 